\title{
Gua Sha, An Asian Traditional Therapy That Could Mimic Physical Injury
}

Young Sam Kim ${ }^{1^{*}}$, Seo Jeong Lee ${ }^{1^{*}}$, Sang A Kim ${ }^{1}$, Kwang Yeon Lee ${ }^{1}$, Seung Pyo Oh${ }^{1}$, Bong Soo Kweon ${ }^{1}$, Young Nam Kwon ${ }^{1}$,

Seong Hwan Park ${ }^{2}$, Jinhyuk Choi ${ }^{2}$

${ }^{1}$ Crime Scene Investigation Unit, Forensic Science Division, Gyeonggi Bukbu Provincial Police, Uijeongbu, Korea, ${ }^{2}$ Department of Forensic Medicine, Korea University College of Medicine, Seoul, Korea

*These authors contributed equally to this work.

Received: January 24, 2022

Revised: February 10, 2022

Accepted: February 23, 2022

\section{Correspondence to}

Seong Hwan Park

Department of Forensic Medicine, Korea University College of Medicine, 73 Inchon-ro, Seongbuk-gu, Seoul 02841 , Korea

Tel: +82-2-3290-1114

Fax: +82-2-928-3901

E-mail:kuforen@gmail.com
Gua Sha is a traditional Asian medicine with different names in many Asian countries. It is a treatment to scrape the petechiae of the skin with ointment. It has generally been administered to patients with upper respiratory infections, heat syndrome, and pain. Herein, we report the case of a 31-year-old Cambodian man who was found dead at a farm accommodation. During the autopsy, multiple linear intradermal hemorrhages without subcutaneous damage were observed in the upper chest and both shoulders. The cause of death was sudden unexpected nocturnal death syndrome because he was a young Asian man who died while sleeping without specific findings. Since it is an unfamiliar traditional medicine in Korea compared to other Asian countries, forensic officers without background knowledge about Gua Sha initially suspected that he was severely assaulted or pressed against a particular object enough to cause crush asphyxia. Because of the difficulty of going to the hospital during the coronavirus disease 2019 pandemic, Gua Sha was used to treat headaches and mild fever. Therefore, this case shows that understanding the culture of other countries could be helpful for investigations especially with the increase of multicultural families in Korea.

Key Words: Traditional medicine; Gua Sha; Petechiae; Crush asphyxia; Sudden unexpected nocturnal death syndrome; Autopsy

\section{Case Report and Discussion}

The deceased was a 31-year-old Cambodian man who was found dead at a farm accommodation in Pocheon city, Gyeonggi-do, on May 26, 2021, around 06:00 AM. The accommodation was a 180-square-foot container with a one-room structure at the entrance of a greenhouse farm in a rural area. The front door is unlocked. According to his colleagues, he usually does not lock his front door. The security camera around the lodge confirmed that he had entered the lodge at around 05:00 PM the previous day. He was wearing a pair of blue sweatpants with no shirt and was found in the supine position looking up at the ceiling (Fig. 1A). He had been suffering from upper respiratory infections, but the postmortem coronavirus disease 2019 test 
result was negative. Some medications, such as acetaminophen and Siang pure balm, were found on top of a nearby drawer. Siang pure balm is an ointment used in folk massage therapy in Cambodia (Fig. 1B). According to the deceased's Cambodian colleague, as the deceased had a headache and mild fever, he was treated with a traditional therapy called Gua Sha. It was determined through photo evidence saved on a colleague's phone that the petechiae on the skin of the deceased had been caused by Gua Sha. Furthermore, there were no other traces, such as bloodstains, suicide notes, or lost items near the accommodation.

The inspection was performed after removing all clothing from the scene. Several petechiae were observed on both sides of the conjunctiva (Fig. 2A). The cornea was transparent. There was no trauma to the head, face, neck, or both the arms and legs. Body fluids leaked from the oral cavity. The tongue protruded from the mouth without signs of trauma. However, a $30 \times 15$ $\mathrm{cm}$ area on the chest and both shoulders had petechiae along with 11 indentations measuring $7.5 \times 1.6 \mathrm{~cm}$ (Fig. 2B). Cyanosis was observed in the fingernails of both hands.

An autopsy was performed to exclude the possibility of violent death due to assault or crush asphyxia. Multiple linear petechiae showed intradermal hemorrhages without subcutaneous hemorrhage on the upper chest and shoulders (Fig. 2C). Because the intradermal hemorrhages were very shallow and the rib cage was not damaged, the possibility of crush asphyxia was ruled out. In addition, no fatal diseases were observed. No other drugs or toxins were found in the toxicological report. Therefore, because he was a healthy young Southeast Asian man who died while sleeping, the cause of death was considered sudden unexpected nocturnal death syndrome, which is thought to be a genetic disorder.

Gua Sha is a traditional Asian therapy in which a tool is used to scrape a patient's skin to treat pain, heatstroke, and upper respiratory disease [1,2]. Gua Sha literally means "to scrape sand," which refers to the petechiae resembling sand after the treatment. These characteristic petechiae were created by closely timed, repeated, unidirectional press-stroking with a smoothedged instrument over a lubricated skin area. The petechiae readily fade into ecchymosis, which resolves entirely within several days. Gua Sha originated from China but has become very popular in other Asian countries [3]. It is referred to by various names in different Asian countries [4]. For example, it is known as ga-sal or kos khyal in Cambodia, cao gio in Vietnam, and khould lam in Laos [5]. However, because it is an uncommon therapy in Korea, it can be misinterpreted as physical damage at the scene of death. Therefore, we aimed to provide basic data for effective investigation through a case report of Gua Sha.

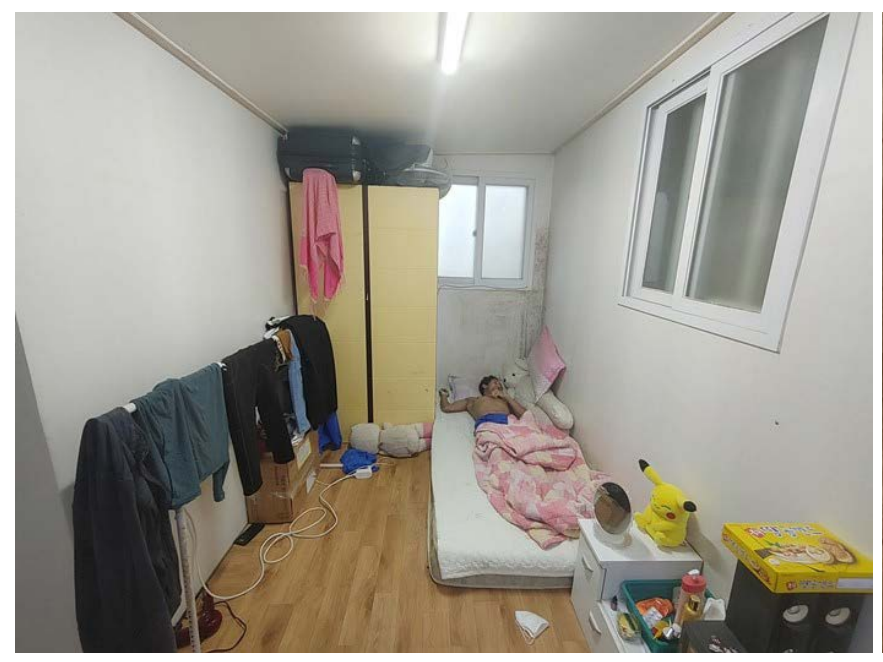

A

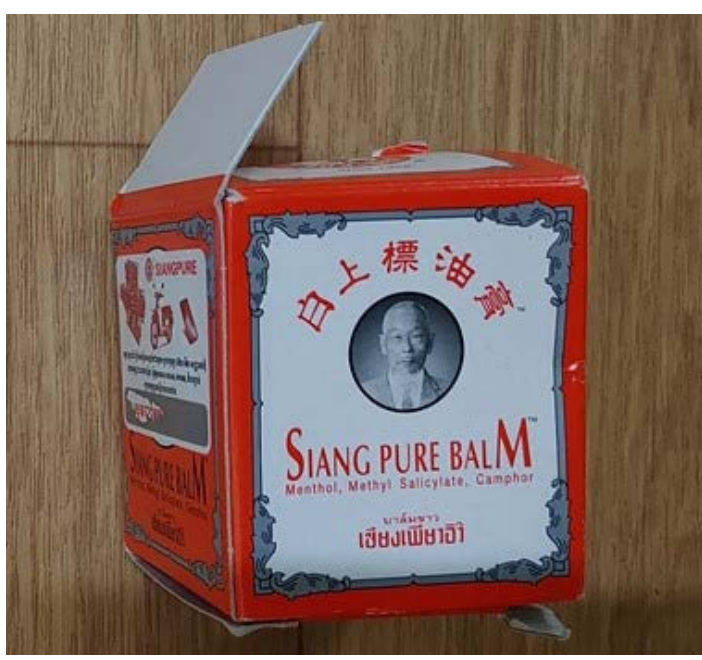

B

Fig. 1. The farm accommodation of the deceased. (A) The deceased lying in a room with a blanket on his lower limbs while lying facing the ceiling. (B) The ointment called Siang pure balm used in Gua Sha. 


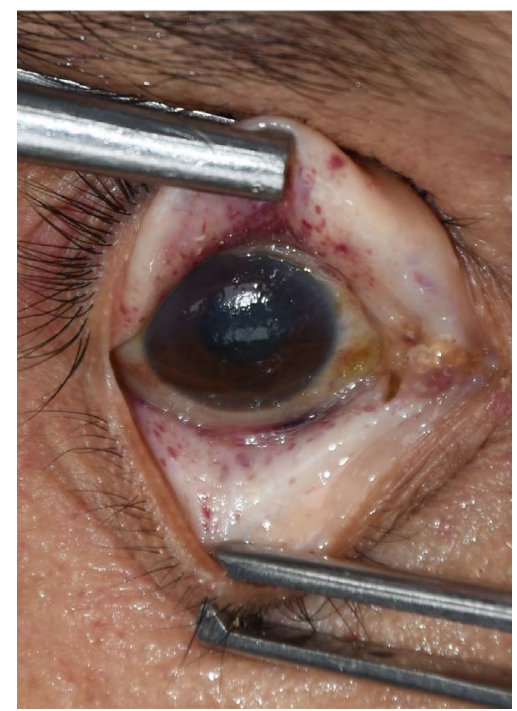

A

Fig. 2. The finding of postmortem inspection and autopsy. (A) The multiple petechiae of the right conjunctiva. (B) The multiple linear intradermal hemorrhages on the upper chest and both shoulders. (C) The Intradermal hemorrhage without subcutaneous hemorrhage on the subcutaneous tissue of upper chest and both shoulders.

Gua Sha is used in various body parts, although the primary locations for application are the posterior thorax, shoulders, chest, temples, and forehead [6]. In this process, marks by the tool remain on the skin, which can be vertically marked along the spine or parallel marks along the ribs which will disappear over time $[7,8]$. These marks can sometimes be mistaken for physical damage, such as abuse and violence, and may be confused with damage caused by crushing objects. Moreover, the coroner and forensic officers at the scene were initially confounded by petechiae in the conjunctiva, which was suspected to be crush asphyxia. As a forensic agent lacking background information, this was an unusual case that required revisiting and further consideration.

Although Gua Sha is a popular folk remedy in some Asian countries [9], there are few reports of Gua Sha in Korean literature. Therefore, various patterns caused by Gua Sha tools should be classified in the future. In addition, it is necessary to summarize the signs caused by folk remedies in other countries because of the increasing number of migrants from various countries.

For forensic officers who need to comprehensively

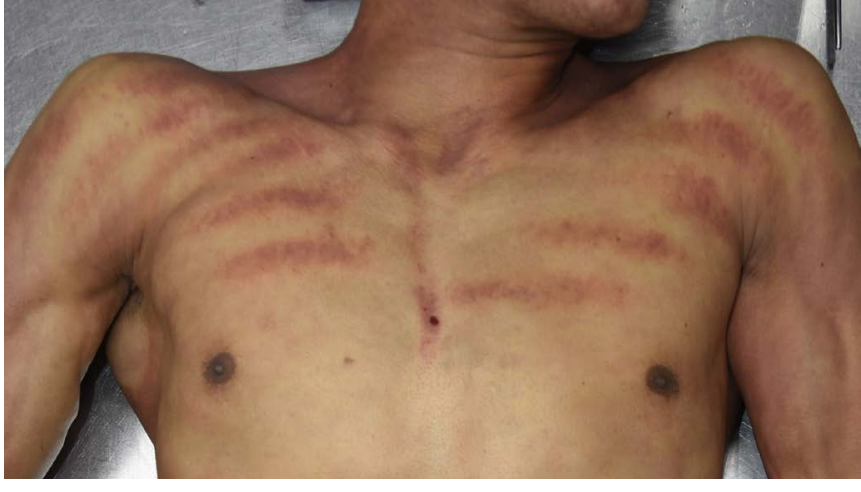

B

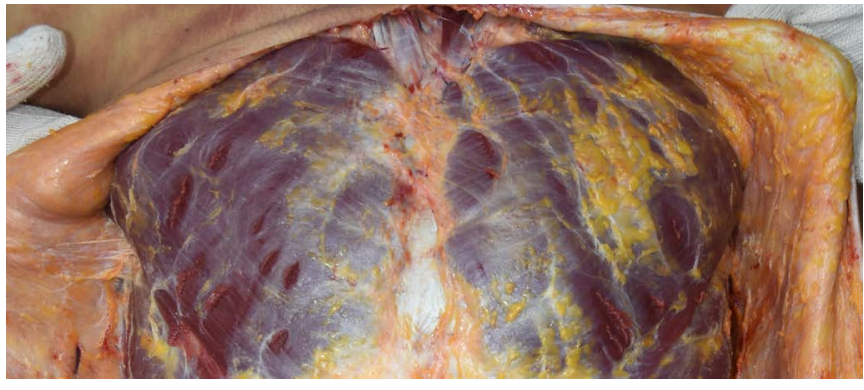

C

investigate the body and the deceased's surroundings for legal judgment of death, it is vital to determine the cause and form of damage present on the body. In similar cases, if in-line bleeding is visible from both shoulders, and the chest of the deceased was determined to have physical damage, it could cause confusion in the investigation process and waste time and human resources. Forensic practice on the performance of death inspection at a crime scene is heavily affected by experiences. Therefore, this case will greatly benefit the decision-making process of forensic officers performing death inspections, which can occur with the increase of multicultural families.

ORCID: Young Sam Kim: https://orcid.org/0000-0003-02660881; Seo Jeong Lee: https://orcid.org/0000-0002-57583679; Sang A Kim: https://orcid.org/0000-0002-0786-6312; Kwang Youn Lee: https://orcid.org/0000-0003-3287-5218; Seung Pyo Oh: https://orcid.org/0000-0001-9535-6293; Bong Soo Kweon: https://orcid.org/0000-0001-9104-5439; Young Nam Kwon: https://orcid.org/0000-0002-2361-668X; Seong Hwan Park: https://orcid.org/0000-0003-2096-2664; Jinhyuk Choi: https://orcid.org/0000-0002-0316-5973 


\section{Conflicts of Interest}

Jinhyuk Choi, a contributing editor of the Korean Journal of Legal Medicine, was not involved in the editorial evaluation or decision to publish this article. All remaining authors have declared no conflicts of interest.

\section{References}

1. Park JH, Shim JW, Cho WY, et al. Literature review of tool-based manipulation for musculoskeletal diseases-with focus on Guasha and IASTM.J Korean Med Rehabil 2016;26:57-65.

2. Nielsen A, Knoblauch NT, Dobos GJ, et al. The effect of Gua Sha treatment on the microcirculation of surface tissue: a pilot study in healthy subjects. Explore (NY) 2007;3:456-66.

3. Liu MY, Chen KJ. Gua Sha, an ancient technical management, for certain illness. Chin J Integr Med 2013;19:3-4.
4. Nielsen A, Kligler B, Koll BS. Safety protocols for gua sha (press-stroking) and baguan (cupping). Complement Ther Med 2012;20:340-4.

5. Nielsen A. Gua sha research and the language of integrative medicine.J Bodyw Mov Ther 2009;13:63-72.

6. Lam NV, Castellanos A, Thompson K. A Vietnamese child with a rash on the back. Am Fam Physician 2016;93:131-2.

7. Aprile A, Pomara C, Turillazzi E. Gua Sha a traditional Chinese healing technique that could mimick physical abuse: a potential issue with forensic implications: a case study. Forensic Sci Int 2015;249:e19-20.

8. Viero A, Amadasi A, Blandino A, et al. Skin lesions and traditional folk practices: a medico-legal perspective. Forensic Sci Med Pathol 2019;15:580-90.

9. Odhav A, Patel D, Stanford CW, et al. Report of a case of Gua Sha and an awareness of folk remedies. Int J Dermatol 2013;52:8923. 